\title{
Competere attraverso il turismo creativo: un'analisi delle preferenze verso la Sicilia ${ }^{1}$
}

\section{Sonia Caterina Giaccone - Marco Galvagno - Elisa Di Mauro}

\begin{abstract}
Obiettivo del paper: Il lavoro studia le preferenze dei potenziali turisti rispetto ad alcune proposte di esperienze turistiche di tipo creativo in Sicilia.

Metodologia: L'indagine esplorativa è stata condotta attraverso la somministrazione di un questionario compilabile on line da turisti italiani e stranieri non residenti in Sicilia. I dati raccolti sono stati elaborati applicando la conjoint analysis e la segmentazione flessibile.

Risultati: I risultati mostrano la preferenza dei turisti per pacchetti che comprendono la più ampia varietà di esperienze (enogastronomiche, ludiche, sportive, culturali), ed in particolare per quelli con almeno un'esperienza di tipo creativo. Inoltre, l'analisi ha permesso di individuare le caratteristiche di un pacchetto ideale.
\end{abstract}

Limiti della ricerca: Il principale limite è rappresentato dal fatto che l'indagine è stata condotta con riferimento ad una sola area geografica.

Implicazioni pratiche: Attraverso la segmentazione della domanda, lo studio offre stimoli utili non solo ad imprese turistiche e tour operator per definire prodotti e strategie competitive, ma anche ai policy maker interessati a formulare politiche di marketing territoriale per la valorizzazione dell'offerta turistica.

Originalità del lavoro: La ricerca arricchisce la letteratura sul turismo creativo analizzando le preferenze dei turisti rispetto alle diverse tipologie di esperienze e alla loro combinazione in pacchetti turistici.

Parole chiave: turismo creativo; conjoint analysis; segmentazione; Sicilia; marketing esperienziale

Purpose of the paper: The paper aims to study the preferences of potential tourists about some proposals of creative touristic experiences in Sicily.

Methodology: The exploratory survey was conducted via an online questionnaire administered to Italian and foreign tourists living outside Sicily. The data collected were analysed by applying the conjoint analysis and flexible segmentation.

Results: The results show the preference of tourists for packages including the widest variety of experiences (food and wine, games, sports, cultural), and in particular for those with at least one experience of a creative kind. Furthermore, the analysis allowed to identify the characteristics of an ideal package.

1 La ricerca alla base del lavoro è stata sviluppata in modo congiunto dagli autori, tuttavia la stesura finale dell' articolo può essere attribuita a Sonia C. Giaccone per i paragrafi 1, 2, 3, 4, 5.1; a Elisa Di Mauro per il paragrafo 5.2; a Marco Galvagno per i paragrafi 5.3 e 6. 
Sinergie

Vol. 35, N. 103, 2017

Research limitations: The main limitation is that the survey was conducted with regard to the tourism of a single geographic area.

Practical implications: Through the demand segmentation, the research provides useful stimulus not only to tourist companies and tour operators in defining their products and competitive strategies, but also to policy makers interested in formulating regional marketing policies to promote tourism.

Originality of the paper: This paper enhances the creative tourism literature analysing the preferences of tourists toward different types of experiences and their combination into tourist packages.

Keywords: creative tourism; conjoint analysis; segmentation; Sicily; experiential marketing

\section{Introduzione}

Questo studio affronta il tema del turismo creativo, fondato sulla capacità di una destinazione di offrire un insieme di attività emozionanti e coinvolgenti, che consentano al turista di esprimere la propria creatività, rendendolo co-creatore della sua vacanza (OECD, 2014). Questa forma di turismo rappresenta un'evoluzione del tradizionale turismo culturale, basato sulle risorse materiali del territorio (come i monumenti e di luoghi di interesse storico ed artistico), e si caratterizza per la valorizzazione delle risorse immateriali di una destinazione (come le tradizioni e la cultura locale) e per il ruolo attivo svolto dal turista.

Il valore del turismo creativo risiede nell'integrazione tra esperienza turistica e contenuti creativi, che consente di raggiungere nuovi segmenti di domanda. A tal proposito, una recente ricerca di TripBarometer (2014) ha evidenziato come il principale desiderio dei turisti sia rappresentato dalla ricerca di esperienze uniche e interessanti, che consentano loro di divertirsi e contemporaneamente di allargare i propri orizzonti calandosi nella vita e nella cultura locale dei luoghi visitati.

Ciò crea l'esigenza di adeguare l'offerta turistica delle destinazioni, arricchendola di esperienze creative, al fine di migliorarne l'immagine e la competitività. In tale direzione, l'UNESCO, già dal 2004, ha lanciato il Creative Cities Network, al fine di rafforzare la cooperazione tra le città che hanno riconosciuto la creatività come fattore strategico di sviluppo (http:// en.unesco.org/creative-cities/).

Sebbene quanto evidenziato denoti la crescente rilevanza del fenomeno, il turismo creativo ha ricevuto una limitata attenzione da parte degli studiosi. In particolare, i lavori esistenti si sono concentrati principalmente sugli aspetti teorici e definitori del fenomeno e sullo studio delle caratteristiche dell'offerta (Richards e Raymond, 2000; Richards, 2011; Richards e Wilson, 2006; Fernandes, 2011; Hull e Sassenberg, 2012), mentre minore attenzione è stata dedicata allo studio del comportamento e delle preferenze della domanda (Tan et al., 2014; Ali et al., 2016). Questo articolo, diversamente da questi ultimi, concentrati principalmente sull' analisi di aspetti psicologici legati alla percezione delle esperienze creative (Tan et al., 2013) e sulla motivazione, la soddisfazione e le intenzioni di 
ritorno presso la destinazione (Chang et al., 2014), si propone di analizzare le preferenze dei turisti rispetto alle diverse tipologie di esperienze creative. In particolare, attraverso un'indagine empirica, il lavoro misura l'utilità attribuita alle diverse tipologie di esperienze ed il gradimento rispetto alla loro combinazione all' interno di pacchetti turistici, individuando infine due segmenti di domanda. La ricerca quindi fornisce spunti di riflessione su alcune opportunità di ridefinizione dell'offerta turistica di tipo creativo, al fine di migliorare la competitività del territorio e delle imprese turistiche.

\section{La customer experience nella letteratura}

L'evoluzione dei modelli di consumo ha innescato un processo di massificazione dei beni, e successivamente anche dei servizi, stimolando la domanda di "esperienze". Ciò è confermato dalla crescente richiesta di emozioni e coinvolgimento alla quale i turisti attribuiscono anche un valore economico progressivamente crescente, che si traduce in una maggiore disponibilità a pagare (Pine e Gilmore, 2000).

Il valore delle esperienze è stato evidenziato nello studio pionieristico di Pine e Gilmore (1998), cui sono seguiti diversi altri contributi che ne hanno enfatizzato l'importanza anche in ambito turistico (Walls et al., 2011; Tung e Ritchie, 2011).

Pine e Gilmore (1998) introducono il tema dell' economia delle esperienze, considerando l'esperienza come una nuova forma di offerta unica. Essa è stata anche considerata come qualcosa di divertente e memorabile (Oh et al. 2007; Tung e Ritchie, 2011) che crea coinvolgimento, interesse e discontinuità rispetto alla vita ordinaria. Nel tempo altri studiosi hanno approfondito diversi aspetti della customer experience: ad esempio, O'Sullivan e Spangler (1998) pongono l'accento sulla partecipazione ed il coinvolgimento dell'individuo, a livello fisico, mentale, emotivo, sociale e spirituale. Sui diversi livelli di coinvolgimento si soffermano anche Gentile et al. (2007), i quali evidenziano la natura olistica e complessa dell' esperienza. Essa si compone di diversi elementi di tipo emozionale, cognitivo, sensoriale, relazionale, valoriale e pratico, la cui interazione determina combinazioni e risultati unici. Gupta e Vajic (1999) interpretano la customer experience come una modalità di acquisizione di conoscenza che si verifica attraverso l'interazione con diversi elementi, tra cui l'ambiente, i beni e i servizi percepiti dal consumatore, in un contesto creato dall'impresa. Verhoef et al. (2009) evidenziano la natura dinamica della customer experience, tenendo conto dell'impatto delle esperienze precedentemente vissute su quelle attuali.

Alcuni elementi ricorrenti che caratterizzano la customer experience possono essere quindi ricondotti: da una parte alla natura olistica, straordinaria e memorabile dell' esperienza, dall' altra all' interazione ed al coinvolgimento dell'individuo a diversi livelli, con gli altri individui e con l'impresa.

L'esperienza è dunque di per sé un prodotto complesso, perché si compone di una molteplicità di elementi, alcuni dei quali direttamente controllabili dall' impresa, come l'atmosfera ed il design del contesto,
Sonia Caterina Giaccone Marco Galvagno Elisa Di Mauro Competere attraverso il turismo creativo: un'analisi delle preferenze verso la Sicilia

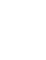


sinergie Vol. 35, N. 103, 2017

la varietà dei prodotti-servizi offerti, le opportunità di interazione tra dipendenti e con i clienti; altri, invece, non controllabili dall impresa, poiché strettamente connessi all' individuo, come la motivazione d' acquisto e l'influenza degli altri (Walls et al., 2011; Verhoef et al., 2009).

L'impresa, in particolare, può intervenire sulle percezioni del cliente esponendolo, durante la fruizione dell' esperienza, ad una molteplicità di stimoli, sia di tipo funzionale (relativi ad esempio alle caratteristiche specifiche dell'offerta) che di tipo emozionale (suscitati da cose o dal comportamento delle persone), che si traducono in impressioni e sensazioni (Carbone e Haeckel, 1994; Garg et al., 2010).

In questa direzione, le principali leve per influenzare le percezioni del cliente possono essere ricondotte alla personalizzazione dell' esperienza, che impone all'impresa la flessibilità necessaria per adeguare tempestivamente la propria offerta specifica alle esigenze della domanda (Pencarelli e Forlani, 2011); alla predisposizione di contesto dove l'esperienza possa essere vissuta, definendo opportunamente elementi come l'atmosfera, i colori, i suoni, per impressionare positivamente le percezioni del cliente (Farias et al., 2014) e alla "messa in scena" dell' esperienza, attraverso lattiva collaborazione dei dipendenti, che, entrando nel personaggio da rappresentare, si comportino come dei veri e propri attori (Maital, 1999).

\section{Definizione e caratteristiche del turismo creativo}

I primi lavori scientifici che hanno affrontato il tema del turismo creativo sono stati pubblicati negli Annals of Tourism Research intorno agli anni Novanta del secolo scorso (Creighton, 1995; Daniel, 1996). In particolare, un numero speciale della rivista nel 1993 è stato dedicato alla produzione di manufatti ed esperienze artistiche (artigianato locale, festival culturali e rappresentazioni teatrali) nell' ambito di attività turistiche (Cohen, 1993; Swain, 1993). Lavori più recenti hanno analizzato il ruolo attivo dei turisti nella co-creazione delle esperienze; ad esempio Gibson e Cornell (2005) hanno studiato il ruolo dei turisti nello sviluppo di forme di spettacoli musicali in giro per il mondo, mentre Buchmann et al. (2010) hanno focalizzato la loro attenzione sull' esperienza dei partecipanti a tour turistici legati alle produzioni cinematografiche (Hudson et al., 2006; Jones e Smith, 2005). Tale processo di co-creazione di esperienze autentiche si manifesta anche in alcune attività turistiche più comuni come il consumo di prodotti alimentari della tradizione locale o la partecipazione ad attività tipiche dei luoghi visitati (Morgan et al., 2008; Prebensen e Foss, 2011).

In sintesi, due sono gli approcci seguiti dai primi studi sul turismo creativo. Il primo si concentra sugli aspetti legati alla produzione e all' offerta ed evidenzia l'esigenza da parte degli operatori e delle destinazioni di differenziarsi dai concorrenti predisponendo attività ed esperienze creative collegate alle tradizioni locali (Hull e Sassenberg, 2012). Il secondo, centrato sulla domanda e sul consumo, analizza le percezioni e le motivazioni che spingono il turista a vivere esperienze uniche e tipiche della destinazione visitata (Tan et al., 2013, 2014; Chang et al., 2014). 
Secondo Richards (2011), il termine "turismo creativo" fu coniato da Pearce e Butler (1993); tuttavia la prima definizione fu quella di Richards e Raymond (2000), in seguito perfezionata dallo stesso Raymond, che nel 2007 definiva il turismo creativo come "una forma più sostenibile di turismo che offre un' atmosfera autentica di cultura locale per il tramite di laboratori informali, pratici e creativi, che si svolgono in piccoli gruppi nelle case e nei luoghi di lavoro, permettendo ai turisti di esplorare la loro creatività, accostandosi alla popolazione locale". (p.145). Tale definizione evidenzia l'opportunità offerta al turista di sviluppare la propria creatività attraverso la co-creazione (Prahalad e Ramaswamy, 2000) e la partecipazione attiva ad esperienze che riflettono i tratti tipici della destinazione e gli consentono di sviluppare un più stretto legame con le usanze, le tradizioni e le persone del luogo (Richards, 2011; Hull e Sassenberg, 2012).

La creatività è dunque un tema centrale nel turismo, sia se riguarda il turista che intende distinguersi ed affermare la propria autenticità, sia se si riferisce agli operatori turistici che, a livello di destinazione, progettano l'offerta turistica e le esperienze da proporre ai viaggiatori.

L'esperienza di viaggio si compone di varie tipologie di attività. In relazione al grado di assorbimento o immersione e di partecipazione attiva o passiva, è possibile distinguere diverse dimensioni, che dovrebbero essere tutte presenti perché l'esperienza sia memorabile: l'intrattenimento, l'esperienza estetica, l'educazione e l'evasione (Pine e Gilmore, 2000). Lambito dell' intrattenimento comprende ad esempio la partecipazione ad uno spettacolo teatrale o l'ascolto di musica, in cui l'individuo non svolge un ruolo attivo ma l'esperienza viene captata e "assorbita" dalla sua mente. L'esperienza estetica, sebbene non implichi la partecipazione attiva del soggetto, ne sollecita le emozioni e ne comporta l'immersione, poiché il soggetto entra dentro l'esperienza e ne viene coinvolto in modo fisico e/o virtuale, come ad esempio avviene in occasione di una visita di un museo o di un'escursione naturalistica. Le esperienze di educazione, quali l' apprendimento di uno studente, implicano un assorbimento della persona ed un suo coinvolgimento attivo necessario per potere accrescere le proprie conoscenze. Infine, l'evasione implica una partecipazione attiva dell'individuo ed una immersione profonda nell' esperienza, come nel caso della partecipazione ad un corso di cucina o ad una vendemmia.

Sebbene il turismo creativo offra al viaggiatore tutte e quattro le tipologie di esperienze richiamate, è evidente come una netta prevalenza sia da attribuire alle componenti di evasione e di educazione (Richards e Raymond, 2001).

A tal fine, occorre da una parte individuare le determinanti ed i livelli della qualità ricercata dai turisti in esperienze di tipo creativo (Brunetti, 1999), poiché da essi dipende il gradimento per i singoli servizi fruiti e la soddisfazione complessiva del viaggio; dall'altra, individuare le azioni da attuare sull'offerta al fine di generare un gap positivo tra qualità percepita, legata ai contenuti ed alle modalità di erogazione del servizio ed interazione con il cliente, e qualità attesa (Brunetti, 1999; Pine e Gilmore 2000). Quest'ultima dipende da una molteplicità di fattori, alcuni dei quali legati al turista stesso, come la motivazione del viaggio, le informazioni detenute e le precedenti esperienze, altri manovrabili dagli operatori turistici, come 
sinergie Vol. 35, N. 103, 2017

il prezzo e l'attività di comunicazione. Un'offerta di turismo creativo di qualità, in grado di garantire una qualità percepita non inferiore a quella attesa, può rappresentare una risorsa distintiva ed inimitabile e pertanto un fattore competitivo per la destinazione (Richards, 2011; Richards e Wilson, 2006 ) e per la sua immagine (Beerli e Martin, 2004). La sfida per le imprese turistiche locali e per i tour operator è quindi quella di riuscire a progettare pacchetti-vacanza con una armoniosa varietà di esperienze coinvolgenti per soddisfare pienamente il turista, rinsaldare il suo legame con il territorio e stimolare la sua propensione a farvi ritorno (Richards, 2011).

\section{Le opportunità competitive del turismo creativo e la progettazione di pacchetti esperienziali}

Nella valorizzazione dell' offerta di turismo creativo di un territorio, un ruolo particolarmente rilevante è svolto dai tour operator. Se le proposte di viaggio sono ben strutturate e contengono servizi ed esperienze di qualità, possono generare un vantaggio competitivo per le imprese del territorio, contribuendo anche al benessere dei residenti (Croce e Perri, 2008).

La progettazione di un pacchetto turistico creativo necessita in primo luogo della conoscenza del sistema delle risorse materiali e immateriali che, evolvendo nel tempo in sinergia con il sistema di relazioni tra gli attori locali, contribuisce a definire l'identità del territorio. Inoltre, un pacchetto creativo deve opportunamente integrare i servizi di base (alloggio, trasporto, ristorazione ed altre attività creative) con alcune esperienze di evasione che, consentendo al turista di vivere dal di dentro il luogo e condividerne emozioni, tradizioni, abitudini e tipicità, contribuiscano a rendere unico e memorabile il ricordo complessivo del viaggio (Kim, 2010; Kim et al., 2010).

Nella definizione di un itinerario occorre individuare un tema conduttore che conferisca unicità all' esperienza, creando il collegamento con il territorio, le sue risorse, le sue storie e tradizioni (Pencarelli e Forlani, 2011). La durata del soggiorno ed il periodo di svolgimento della vacanza sono elementi importanti da definire, che influenzano a loro volta la lunghezza/articolazione del percorso, il mezzo di trasporto da selezionare ed il numero di tappe e soste da effettuare (Croce e Perri, 2008). Un altro fattore critico è la scelta dei luoghi da visitare (musei, monumenti) e delle esperienze da proporre. In particolare, due criteri guida per selezionare gli operatori dell'offerta da coinvolgere in pacchetti turistici creativi (come ad esempio le cantine dove organizzare degustazioni, le aziende alimentari dove svolgere lezioni di cucina, le aziende agricole in cui partecipare alla vendemmia) sono: l'accessibilità (sia virtuale, attraverso il sito web e la comunicazione sui social network, sia in senso fisico, legata ad esempio all' esistenza di segnaletica stradale, illuminazione e disponibilità di aree di parcheggio) e l' accoglienza. Quest'ultima si riferisce alle caratteristiche degli ambienti, da quelli di produzione a quelli utilizzati per la degustazione o la commercializzazione, in cui il turista può seguire la materia prima dal campo (o dall'allevamento), osservare e partecipare ad alcuni processi 
di lavorazione, assaggiare il prodotto finito ed, infine, avere la possibilità di acquistarlo; ma soprattutto alla professionalità e alla capacità di coinvolgimento del personale che accoglie e accompagna i turisti durante la permanenza in azienda, aiutandoli a comprendere i processi e ad apprezzare i prodotti realizzati, all' interno di un ambiente accogliente ed amichevole (Croce e Perri, 2008).

\section{Un' analisi empirica delle preferenze dei turisti in Sicilia}

\subsection{Obiettivi e metodologia della ricerca}

L'obiettivo della ricerca è quello di indagare le preferenze di potenziali turisti rispetto ad alcune proposte di pacchetti esperienziali-creativi in Sicilia. Ciò al fine di comprendere, da una parte il gradimento complessivo di pacchetti turistici caratterizzati da diverse tipologie di attività, dall'altra l'utilità attribuita alle specifiche esperienze ed ai livelli in cui tali esperienze possono essere proposte.

L'analisi è di tipo esplorativo e intende rispondere a tre principali domande di ricerca.

D1: Quali sono le preferenze dei viaggiatori rispetto ad alcuni pacchetti turistici esperienziali-creativi?

D2: Qual è l' importanza riconosciuta dai viaggiatori alle singole componenti presenti nei pacchetti turistici esperienziali-creativi?

D3: Quale utilità è associata dai turisti ai livelli alternativi previsti per ciascuna componente del pacchetto?

Le tre domande di ricerca sono consequenziali tra loro e presentano un crescente grado di analiticità. Con la prima si intendono indagare le preferenze dei turisti rispetto a differenti offerte integrate, comprendenti sia elementi tradizionali (come albergo e prezzo) che esperienzialicreativi, rispettando così la natura omnicomprensiva delle forme di turismo creativo (Richards, 2011). Essa mira a conoscere il giudizio di gradimento dei potenziali turisti sulla vacanza nel suo complesso. La seconda domanda di ricerca riguarda nello specifico l'importanza attribuita a ciascuna componente (es. esperienza enogastronomica) dei pacchetti proposti, con l'intento di capire quanta importanza i potenziali viaggiatori diano ai contenuti esperienziali e/o creativi. Infine, la terza domanda di ricerca intende comprendere l'utilità associata alle diverse scelte all'interno di ciascuna tipologia di esperienza (es. lezione di cucina tipica, partecipazione alla vendemmia, visita in cantina con degustazione).

L'indagine è stata condotta attraverso la somministrazione di un questionario ad un campione di potenziali turisti interessati ad un viaggio in Sicilia e la successiva analisi delle valutazioni fornite su alcuni ipotetici pacchetti turistici esperienziali-creativi.

Premesso che la valutazione di un pacchetto turistico da parte del cliente avviene attraverso un processo mentale in cui l'utilità complessiva è funzione della combinazione delle utilità attribuite alle singole componenti, e che il cliente percepisce il pacchetto nel suo insieme, senza riuscire ad esplicitare l'utilità attribuita a ciascuna di esse, si è scelto di
Sonia Caterina Giaccone

Marco Galvagno

Elisa Di Mauro

Competere attraverso

il turismo creativo: un'analisi delle preferenze verso 
sinergie italian journal of management Vol. 35, N. 103, 2017

utilizzare la tecnica della conjoint analysis, che consente di determinare l'utilità associata alle singole componenti di un prodotto, attraverso una logica di scomposizione della sua valutazione complessiva (Furlan e Martone, 2011). Tale tecnica permette, altresì, di pervenire a valutazioni complessive su pacchetti alternativi non sottoposti al giudizio dei clienti, consentendo così di individuare le caratteristiche di un possibile pacchetto ideale o l'utilità di componenti non sottoposte a valutazione.

Infine, sui risultati della conjoint analysis è stata svolta un'analisi di segmentazione, che ha consentito di identificare due segmenti di potenziali turisti in Sicilia, caratterizzati da esigenze e comportamenti differenti.

\subsection{La struttura del questionario}

La raccolta dei dati è stata fatta attraverso la somministrazione di un questionario, compilabile on line e disponibile in lingua italiana ed inglese, ad un campione di 228 turisti italiani e stranieri non residenti in Sicilia.

Il questionario si articola in tre parti principali. La prima rileva alcune caratteristiche comportamentali dei rispondenti, ad esempio: l'importanza attribuita a fattori come "arte e cultura", "esperienze enogastronomiche", "divertimento" e "sport" nella scelta della destinazione in cui trascorrere una vacanza; le modalità di acquisizione delle informazioni (internet, telefono, di persona), di organizzazione della vacanza (fai da te o viaggio organizzato); le preferenze sulla compagnia in viaggio (da soli, in coppia, con amici); la frequenza, la durata media dei viaggi e il periodo preferito. La seconda parte propone tredici pacchetti turistici, di tipo esperienzialecreativo, rispetto a cui i rispondenti sono stati chiamati ad esprimere una valutazione complessiva. La terza parte identifica alcuni caratteri sociodemografici dei rispondenti (genere, età, residenza, professione, struttura familiare) e la volontà degli stessi di effettuare un viaggio in Sicilia.

Le principali componenti inserite nei pacchetti sono sette, ciascuna delle quali è stata articolata in tre livelli (Fig. 1).

Fig. 1: Le componenti dei pacchetti esperienziali-creativi proposti nel questionario

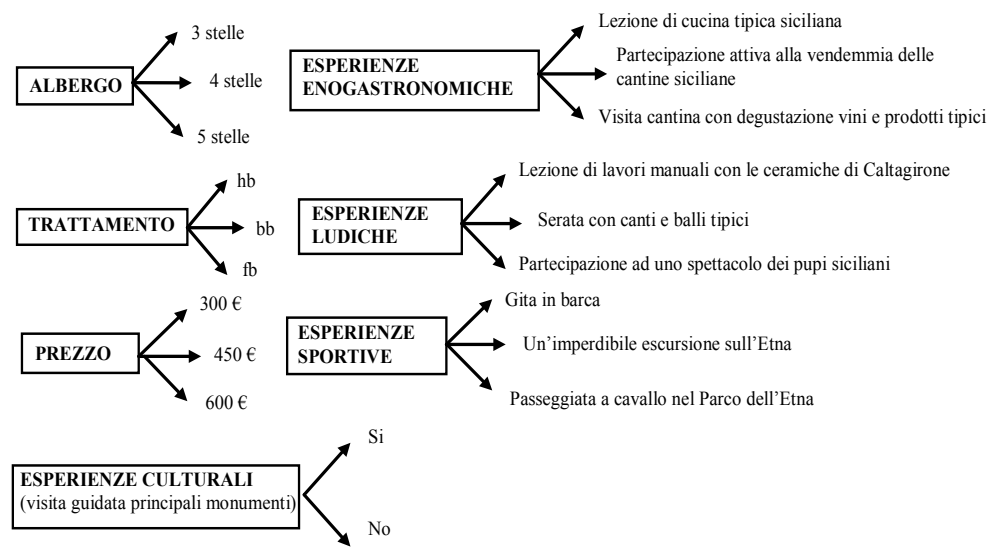

Fonte: Ns. elaborazione 
I pacchetti possono dunque presentare diverse combinazioni di: Sonia Caterina Giaccone albergo a tre, quattro o cinque stelle; trattamento di mezza pensione $(\mathrm{HB})$,

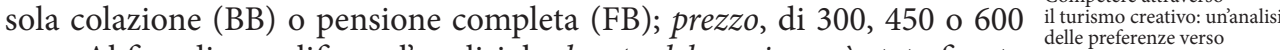
Marco Galvagno euro. Al fine di semplificare l'analisi, la durata del soggiorno è stata fissata la Sicilia in quattro giorni e tre notti per tutti i pacchetti.

Per quanto riguarda le esperienze proposte (di tipo enogastronomico, ludico, sportivo e culturale), le specifiche attività inserite nei pacchetti sono state individuate tenendo conto della classificazione concettuale delle esperienze fornita da Pine e Gilmore (2000) e dei successivi contributi della letteratura relativi alla loro operazionalizzazione (Oh et al., 2007; Kim, 2014; Tan et al., 2013; Ali et al., 2016), i quali hanno individuato le componenti di ciascuna tipologia di esperienza. Sulla base di ciò, e con il contributo di alcuni tour operator, sono state selezionatele attività da inserire nei pacchetti da sottoporre agli intervistati, tenendo in considerazione, da una parte la reale disponibilità nell'offerta turistica del territorio, dall'altra la necessità di assicurare, all'interno dei pacchetti, un mix delle diverse tipologie di esperienze. Quindi, tra le esperienze enogastronomiche sono stati proposti corsi di cucina tipica, visite in cantina con degustazione, partecipazione alla vendemmia; tra le esperienze ludiche vi sono lezioni di lavori manuali, serate con canti e balli tipici o spettacoli folkloristici. Le esperienze sportive includono una gita in barca, un' escursione sull' Etna o una passeggiata a cavallo; infine, le esperienze culturali, quali visite guidate ai principali monumenti e musei, sono state inserite soltanto in alcuni pacchetti, per verificare se ci sia un interesse verso i pacchetti comprensivi anche di tali attività non configurabili tipicamente come esperienze di tipo creativo.

Tab. 1: Le tipologie di esperienze proposte nei pacchetti turistici

\begin{tabular}{|l|c|c|}
\hline \multirow{4}{*}{$\begin{array}{l}\text { Esperienze } \\
\text { enogastronomiche }\end{array}$} & ATTIVITA' PREVISTE & TIPOLOGIA ESPERIENZE \\
\cline { 2 - 3 } & $\begin{array}{c}\text { Lezione di Cucina tipica siciliana } \\
\text { Partecipazione attiva alla vendemmia } \\
\text { nelle cantine siciliane }\end{array}$ & Evasione \\
\cline { 2 - 4 } & $\begin{array}{c}\text { Visita in cantina con degustazione } \\
\text { vini e prodotti tipici }\end{array}$ & Educazione/Evasione \\
\hline \multirow{3}{*}{ Esperienze ludiche } & $\begin{array}{c}\text { Lezione di lavori manuali con le } \\
\text { ceramiche di Caltagirone }\end{array}$ & Evasione \\
\cline { 2 - 4 } & Serata con canti e balli tipici & Intrattenimento \\
\cline { 2 - 4 } Esperienze sportive & Spettacolo dei pupi siciliani & Intrattenimento \\
\cline { 2 - 4 } & $\begin{array}{c}\text { Gita in barca } \\
\text { Escursione sull'Etna }\end{array}$ & Estetica/Educazione \\
\cline { 2 - 4 } & $\begin{array}{c}\text { Passeggiata a cavallo nel parco dell' } \\
\text { Etna }\end{array}$ & Evasione \\
\hline \multirow{2}{*}{ Esperienza culturale } & $\begin{array}{c}\text { Visita dei principali } \\
\text { monumenti }\end{array}$ & Educazione/Estetica \\
\hline
\end{tabular}

Fonte: Ns. elaborazione

Utilizzando il software SPSS, si è ricavato il disegno ortogonale contenente diciotto card, dalle quali, al fine di eliminare ripetizioni e prezzi fuorvianti, sono state selezionate le tredici incluse nel questionario. Gli 
sinergie

italian journal of management Vol. 35, N. 103, 2017

elementi che compongono le 13 card sono sinteticamente riportati nella tabella seguente.

Tab. 2: I pacchetti inseriti nel questionario

\begin{tabular}{|l|c|c|c|c|c|c|}
\hline Albergo & $\begin{array}{c}\text { Prezzo } \\
\text { (euro) }\end{array}$ & $\begin{array}{c}\text { Esperienza } \\
\text { enogastronomica }\end{array}$ & $\begin{array}{c}\text { Esperienza } \\
\text { ludica }\end{array}$ & $\begin{array}{c}\text { Esperienza } \\
\text { sportiva }\end{array}$ & Tratt.to & Card \\
\hline 5 stelle & 450 & lezione di cucina & spettacolo pupi & Etna & BB & 1 \\
\hline 4 stelle & 450 & vendemmia & lavori manuali & Etna & FB & 2 \\
\hline 3 stelle & 300 & vendemmia & lavori manuali & cavallo & BB & 3 \\
\hline 4 stelle & 300 & lezione di cucina & spettacolo pupi & cavallo & BB & 4 \\
\hline 3 stelle & 450 & vendemmia & spettacolo pupi & cavallo & FB & 5 \\
\hline 5 stelle & 600 & visita cantina & canti tipici balli & cavallo & FB & 6 \\
\hline 4 stelle & 300 & visita cantina & lavori manuali & gita barca & BB & 7 \\
\hline 4 stelle & 450 & vendemmia & canti tipici balli & Etna & HB & 8 \\
\hline 5 stelle & 600 & vendemmia & spettacolo pupi & gita barca & HB & 9 \\
\hline 5 stelle & 450 & visita cantina & lavori manuali & cavallo & HB & 10 \\
\hline 4 stelle & 450 & lezione di cucina & lavori manuali & gita barca & HB & 11 \\
\hline 4 stelle & 300 & visita cantina & canti tipici balli & Etna & HB & 12 \\
\hline 4 stelle & 450 & visita cantina & spettacolo pupi & gita barca & FB & 13 \\
\hline
\end{tabular}

Fonte: Ns. elaborazione

Le diverse combinazioni di pacchetti sono state presentate sotto forma di locandine dai colori vivaci, utilizzando immagini rappresentative per identificare una stessa attività contenuta in più card; ciò al fine di aiutare il rispondente ad individuare rapidamente le alternative già a livello visivo. Tutte le locandine presentano, nel medesimo ordine, le componenti del pacchetto: prima l'albergo, poi il trattamento, le esperienze culturali, enogastronomiche, sportive, ludiche e, in posizione centrale, il prezzo. Gli intervistati hanno espresso per ciascuna locandina un giudizio di gradimento su una scala da 1 (non mi piace assolutamente) a 9 (mi piace tantissimo).

Il questionario è stato divulgato utilizzando un metodo snowball, al fine di raggiungere quanti più soggetti in Italia ed all' estero, purché non siciliani. Delle 228 risposte pervenute, 193 sono state quelle del tutto complete e dunque utilizzabili ai fini dell'analisi.

\subsection{I risultati dell' indagine}

\subsubsection{Il profilo dei rispondenti e le preferenze di viaggio}

Il campione di intervistati è composto da 128 femmine e 65 maschi; in prevalenza si tratta di soggetti tra i 45 e i 64 anni, tuttavia è rilevante anche la fascia dei 35-44 anni; 115 di essi hanno figli. La maggior parte dei rispondenti ( $98 \%$ circa) ha mostrato interesse verso la possibilità di fare una vacanza in Sicilia.

I rispondenti provengono quasi esclusivamente dall' Europa (96\%) e risiedono per la maggior parte in Italia (62, 2\%), Regno Unito $(12,4 \%)$ e Germania (7, 3\%).

Per quanto concerne la situazione occupazionale, la maggior parte ha dichiarato di lavorare come dipendente $(46,1 \%)$ o come libero professionista (28\%). 
Con riferimento alle abitudini di viaggio, la tabella seguente mostra media e deviazione standard per le domande in questione, alle quali sono state attribuite valutazioni su una scala da 1 a 9 .

Tab. 3: Le abitudini di viaggio dei rispondenti

\begin{tabular}{|c|c|c|c|}
\hline DOMANDE & ALTERNATIVE & MEDIA & DEV.STD \\
\hline \multirow{4}{*}{$\begin{array}{l}\text { Quanto influiscono queste variabili nella scelta } \\
\text { di una destinazione? }\end{array}$} & Cultura & 7,70 & 1,559 \\
\hline & Enogastronomia & 6,42 & 2,150 \\
\hline & Divertimento & 5,99 & 2,173 \\
\hline & Sport & 4,17 & 2,635 \\
\hline \multirow{4}{*}{$\begin{array}{l}\text { Quanto influiscono queste variabili nella scelta } \\
\text { di una vacanza in Sicilia? }\end{array}$} & Cultura & 7,86 & 1,600 \\
\hline & Enogastronomia & 7,13 & 2,111 \\
\hline & Divertimento & 5,79 & 2,341 \\
\hline & Sport & 3,97 & 2,601 \\
\hline \multirow{7}{*}{ Organizzazione vacanza } & Info web & 7,33 & 2,208 \\
\hline & Info telefono & 5,17 & 2,690 \\
\hline & Fai da te & 6,11 & 2,610 \\
\hline & Tour operator & 4,72 & 2,698 \\
\hline & Prenota sul sito & 5,67 & 2,689 \\
\hline & Prenota con email & 5,80 & 2,680 \\
\hline & Prenota al telefono & 5,01 & 2,870 \\
\hline \multirow{3}{*}{ Durata della vacanza } & Weekend & 5,72 & 2,877 \\
\hline & Settimana & 6,61 & 2,469 \\
\hline & Più di una Settimana & 5,48 & 3,151 \\
\hline \multirow{4}{*}{ Periodo della vacanza } & Dicembre-Febbraio & 4,32 & 2,805 \\
\hline & Marzo-Maggio & 5,93 & 2,693 \\
\hline & Giugno-Agosto & 6,63 & 2,740 \\
\hline & Settembre-Novembre & 5,92 & 2,750 \\
\hline
\end{tabular}

Fonte: Ns. elaborazione

Le prime due domande sulle variabili che influiscono nella scelta di una destinazione mostrano la prevalenza della cultura, dell'enogastronomia e del divertimento, mentre minore importanza è riservata allo sport.

Per quanto riguarda le modalità di organizzazione della vacanza, la maggior parte dei rispondenti dichiara che, nella fase iniziale della ricerca di informazioni, preferisce utilizzare Internet piuttosto che contatti telefonici o personali; inoltre non mostra evidenti preferenze tra il ricorso ad operatori specializzati (tour operator/agenzie di viaggio) e l'approccio fai dai te. Non si rileva una preferenza marcata neanche sulle modalità di prenotazione: sebbene la più apprezzata sia quella tramite email o ricorrendo alla sezione "prenota" del sito web di interesse, non distanti nelle preferenze sono i contatti telefonici e di persona.

Il $23 \%$ ha dichiarato di preferire un periodo di vacanza più lungo di una settimana. Il periodo preferito per le partenze è quello estivo tra giugno e agosto, sebbene anche i periodi primaverili ed autunnali riscuotano apprezzamento.

Dall'analisi delle risposte per fasce di età dei rispondenti sono emerse alcune differenze nelle abitudini e nelle preferenze rispetto alla vacanza. Per i turisti fino ai 24 anni (prevalentemente studenti), il divertimento riveste un' importanza decisiva nella scelta della destinazione, mentre
Sonia Caterina Giaccone Marco Galvagno Elisa Di Mauro Competere attraverso il turismo creativo: unanalisi delle preferenze verso la Sicilia 
sinergie Vol. 35, N. 103, 2017

meno rilevanti sono la cultura, l'enogastronomia e lo sport. Con specifico riferimento ad una vacanza in Sicilia, cresce il valore medio riconosciuto al fattore enogastronomico. L'organizzazione è principalmente "fai da te"; i giovani preferiscono muoversi con gli amici (per gli italiani è ricorrente il viaggio in coppia) ed organizzare la vacanza tramite l'uso del web e delle email per le prenotazioni.

La fascia di età compresa tra i 25 e i 44 anni attribuisce elevata importanza alla cultura e all' enogastronomia, ricerca informazioni su internet e preferisce organizzare la vacanza in maniera autonoma prenotando via email. I viaggiatori si muovono generalmente in coppia o con amici, la maggior parte senza figli.

Per quanto riguarda la fascia di età tra i 45 e i 64 anni, le valutazioni sulle determinanti per la scelta di una destinazione (la Sicilia in particolare) sono analoghe a quelle delle altre fasce. Cambiano piuttosto le modalità di organizzazione del viaggio, non tanto per quanto attiene la raccolta di informazioni, quanto per il venir meno del gap nelle preferenze tra "fai da te" e viaggio organizzato. Le vacanze vengono fatte in prevalenza in famiglia $o$ in coppia, in genere sono della durata di una settimana o più ed il periodo preferito è quello di giugno-agosto. Importanti cambiamenti sulle modalità di organizzazione della vacanza si evidenziano invece per il gruppo degli over 65, che mostra di preferire il viaggio organizzato dal tour operator. Le vacanze sono prevalentemente svolte con la famiglia e con amici per una settimana o anche più ed il periodo preferito è quello tra settembre e novembre.

\subsubsection{La risposta di gradimento verso i pacchetti esperienziali-creativi}

Con riferimento alla valutazione sui 13 pacchetti proposti nel questionario, rispetto ai quali gli intervistati sono stati chiamati ad esprimere complessivamente il loro gradimento su una scala da 1 a 9, la tabella seguente sintetizza le risposte evidenziando i valori medi, la mediana, la moda e la deviazione standard ricavati, considerando le risposte in maniera aggregata.

Tab. 4: Valutazione dei pacchetti turistici esperienziali-creativi

\begin{tabular}{|l|c|c|c|c|}
\hline PACCHETTI & MEDIA & MEDIANA & MODA & DEV. STD \\
\hline Card 1 & 6,59 & 7 & 8 & 2,072 \\
\hline Card 2 & 6,06 & 6 & 7 & 2,150 \\
\hline Card 3 & 6,17 & 7 & 7 & 2,224 \\
\hline Card 4 & 5,99 & 6 & 7 & 2,143 \\
\hline Card 5 & 5,74 & 6 & 6 & 2,126 \\
\hline Card 6 & 5,45 & 6 & 5 & 2,402 \\
\hline Card 7 & 7,10 & 8 & 8 & 1,782 \\
\hline Card 8 & 6,14 & 7 & 7 & 2,111 \\
\hline Card 9 & 5,68 & 6 & 7 & 2,224 \\
\hline Card 10 & 5,64 & 6 & 6 & 2,006 \\
\hline Card 11 & 6,21 & 7 & 7 & 2,092 \\
\hline Card 12 & 6,37 & 7 & 8 & 2,308 \\
\hline Card 13 & 6,44 & 7 & 7 & 2,010 \\
\hline
\end{tabular}

Fonte: Ns. elaborazione 
Le valutazioni medie dei pacchetti variano tra 5 e 7 , ma i più apprezzati sono quelli omnicomprensivi, che presentano, oltre alle componenti esperienziali-creative, quella culturale (assente nei pacchetti n. 2, 4, 10). Il pacchetto preferito è il settimo, che registra un gradimento medio pari a 7 , 10 , moda e mediana pari ad 8 e valori di deviazione standard più contenuti, rispetto anche alle altre soluzioni.

Fig. 2: Pacchetto esperienziale preferito dal campione - Card 7

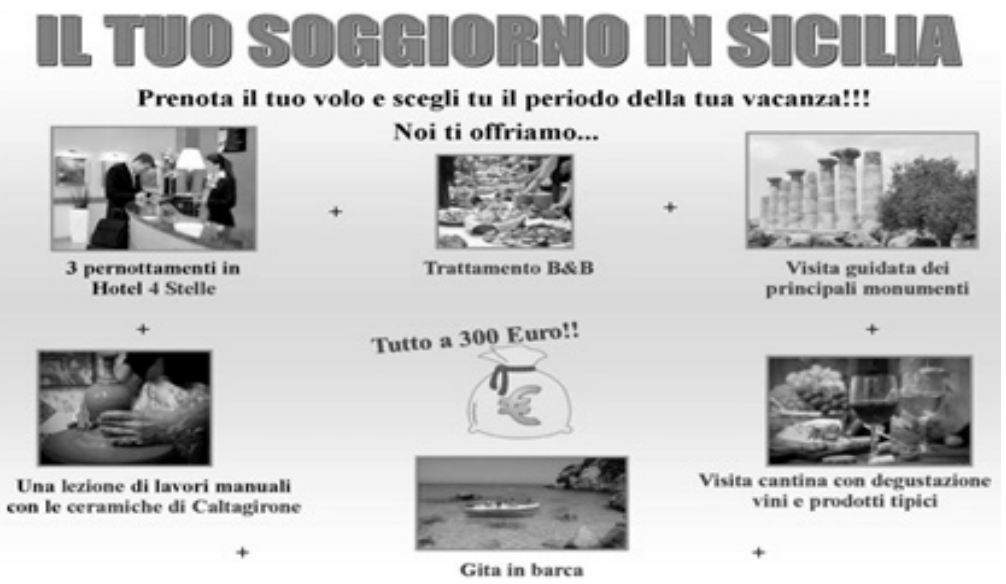

Fonte: Ns. elaborazione

La componente creativa di questo pacchetto propone una "visita in cantina con degustazione" come esperienza enogastronomica, la "lezione di lavori manuali" come esperienza ludica e una "gita in barca" come esperienza sportiva. Il pacchetto propone altresì un' esperienza culturale di tipo tradizionale consistente nella visita guidata ai principali monumenti.

Hanno riscosso elevato gradimento anche i pacchetti $1,3,8,11$, 12, 13, caratterizzati da una combinazione di diverse esperienze (enogastronomiche, ludiche, sportive e culturali) e dalla presenza di attività creative (corso di cucina, lavori manuali, partecipazione alla vendemmia ecc.), che realizzano il massimo coinvolgimento del turista.

Quanto emerso è coerente con il pensiero di Richards (2011), secondo il quale il viaggiatore riconosce utilità ad offerte turistiche caratterizzate dalla presenza di attività creative.

\subsubsection{I risultati della conjoint analysis: valore degli attributi e stima delle utilità}

La conjoint analysis applicata alle valutazioni dei pacchetti consente di stabilire l' importanza riconosciuta a ciascuna componente del pacchetto ed il livello preferito della stessa.

Il prezzo emerge come la componente più rilevante (circa il 24\%), pertanto si ritiene possa svolgere un ruolo critico nella scelta del pacchetto, sebbene, proprio in virtù della differenziazione e della personalizzazione dei servizi proposti, l' applicazione di un prezzo più elevato sia generalmente
Sonia Caterina Giaccone Marco Galvagno Elisa Di Mauro

il turismo creativo: un'analisi delle preferenze verso 
sinergie Vol. 35, N. 103, 2017

accettata dai clienti. Pertanto, pur consapevoli che la competitività nel business del turismo creativo non possa essere perseguita attraverso una politica di prezzi al ribasso, tale risultato fa comunque riflettere sulla necessità di riuscire a trovare un equilibrio nel prezzo tra l' offerta e la tipologia di domanda.

Gli altri fattori osservati, specificamente il trattamento (17\%), l'esperienza sportiva (16)\%, le esperienze enogastronomica, ludica e la categoria di albergo (14\%) non presentano elevati scostamenti nelle valutazioni e appaiono dunque rilevanti in modo simile nella composizione di un pacchetto turistico esperienziale-creativo. Perciò, diventa critico il contributo dell'operatore turistico nel selezionare opportunamente le diverse esperienze per la composizione di un pacchetto competitivo.

Una volta evidenziata l' importanza di ciascun attributo del pacchetto, l' analisi è stata approfondita mostrando quale livello di ciascuno di essi fosse maggiormente gradito ai rispondenti.

Nell' ambito dell' esperienza enogastronomica, la "visita in cantina" è preferita rispetto alla "lezione di cucina tipica siciliana" ed alla "partecipazione attiva alla vendemmia". Dell' esperienza ludica è stato espresso un maggiore gradimento per i "lavori manuali", seguiti dallo "spettacolo dei pupi siciliani" e dalla "serata con canti e balli tipici". Nell'ambito dell' esperienza sportiva si rileva una preferenza per "l'escursione sull'Etna" e per la "gita in barca", rispetto alla "passeggiata a cavallo". Il trattamento preferito risulta il "b\&b" seguito dalla "pensione completa" e dalla "mezza pensione". La categoria preferita è il "3 stelle", seguita dal "4 stelle" e dal " 5 stelle".

Tab. 5: Stima delle utilità

\begin{tabular}{|l|l|l|l|}
\hline & & Stima di utilità & Errore std. \\
\hline \multirow{5}{*}{ Esperienza enogastronomica } & corso di cucina & $-0,112$ & 0,164 \\
\cline { 2 - 4 } & vendemmia & $-0,118$ & 0,124 \\
\cline { 2 - 4 } & visita in cantina & 0,230 & 0,207 \\
\hline \multirow{5}{*}{ Esperienza ludica } & lavori manuali & 0,049 & 0,112 \\
\cline { 2 - 4 } & canti e balli tipici & $-0,077$ & 0,191 \\
\cline { 2 - 4 } & spettacolo pupi & 0,028 & 0,146 \\
\hline \multirow{5}{*}{ Trattamento } & gita in barca & 0,191 & 0,168 \\
\cline { 2 - 4 } & escursione sull Etna & 0,257 & 0,167 \\
\cline { 2 - 4 } & passeggiata a cavallo & $-0,447$ & 0,096 \\
\hline Categoria albergo & bed \& breakfast & 0,415 & 0,242 \\
\cline { 2 - 4 } & mezza pensione & $-0,244$ & 0,113 \\
\cline { 2 - 4 } & pensione completa & $-0,171$ & 0,212 \\
\hline \multirow{5}{*}{ Prezzo } & 3 stelle & $-0,174$ & 0,231 \\
\cline { 2 - 4 } & 4 stelle & $-0,347$ & 0,461 \\
\cline { 2 - 4 } & 5 stelle & $-0,521$ & 0,692 \\
\hline \multirow{5}{*}{ (Costante) } & 300 Euro & $-0,025$ & 0,358 \\
\cline { 2 - 4 } & 450 Euro & $-0,049$ & 0,358 \\
\cline { 2 - 4 } & 600 Euro & $-0,074$ & 0,716 \\
\hline & & 6,543 & 0,329 \\
\hline
\end{tabular}

* I valori inseriti nella colonna "stima di utilitä" non vanno intesi come valori assoluti, ma come valori relativi.

Fonte: ns. elaborazione 
Attraverso le stime di utilità, è stato possibile individuare le caratteristiche del pacchetto ideale, che prevede una visita in cantina come esperienza enogastronomica, lo svolgimento di lavori manuali come esperienza ludica e un' escursione sull'Etna, come esperienza sportiva. La Sonia Caterina Giaccone Marco Galvagno Elisa Di Mauro Competere attraverso il turismo creativo: un'analisi delle preferenze verso categoria di albergo preferita è il 3 stelle con trattamento b\&b, al prezzo di 300 euro.

Quanto evidenziato, tuttavia, non sarebbe completo se non si considerassero insiemel'importanza relativa e lo scarto di utilità per ciascun attributo. In particolare, il fatto che il prezzo abbia l'importanza relativa maggiore, ma che lo scarto tra le utilità dei differenti livelli sia molto basso rispetto a quello degli altri fattori, indica che, seppur esso sia considerato importante nella valutazione del pacchetto, la differenza tra il prezzo più basso e quello più alto non è poi così elevata e non esiste una differenza netta tra i rispondenti con riferimento a tale attributo. Lo sport, al contrario, ha un'importanza relativa media, ma presenta un elevato scarto di utilità tra le differenti alternative. In questo caso esiste una notevole differenza tra i rispondenti per quanto riguarda l'utilità assegnata a ciascuna alternativa, quindi occorrerà riservare particolare attenzione nella scelta delle attività sportive del pacchetto, in quanto, allontanandosi dalla soluzione preferita, si rischia di soddisfare solo una parte dei clienti e lasciare insoddisfatti gli altri. Alla luce di quanto detto, le variabili con il più ampio scarto di utilità (nel nostro caso l'esperienza sportiva e quella ludica) sono le più delicate da gestire, poiché una scelta inopportuna dell'alternativa rischia di non offrire la massima utilità al turista. In riferimento alle alternative con il maggior scarto di utilità, dunque, sarebbe preferibile segmentare la clientela ed offrire pacchetti differenziati.

\subsubsection{La segmentazione dei potenziali turisti}

L' utilizzo della cluster analysis ha consentito di segmentare i rispondenti rispetto alle preferenze espresse nei confronti dei pacchetti alternativi. In particolare, sono stati individuati due segmenti di potenziali turisti, il cluster 1 composto da 93 soggetti e denominato "tradizionalisti", il cluster 2 composto da 100 soggetti e denominato come "moderni". Applicando nuovamente la conjoint analysis a ciascuno dei segmenti individuati, è stato possibile indagare le soluzioni preferite da ciascuno di essi.

Per il cluster dei tradizionalisti, il pacchetto ideale comprende la "visita in cantina", lo "spettacolo dei pupi siciliani", l' "escursione sull'Etna" e il soggiorno in hotel " 3 stelle" con trattamento "b\&b" al prezzo di "600 euro".

Per il cluster dei moderni, il pacchetto ideale è composto dalla "visita in cantina", dai "lavori manuali", dal' "escursione sull' Etna" e dal soggiorno in un albergo " 5 stelle" con trattamento "b\&b" al prezzo di "300 euro".

Ciò che emerge è che, mentre il segmento dei tradizionalisti mostra disponibilità a pagare di più senza avanzare pretese sulla categoria dell'alloggio, quello dei moderni, invece, preferisce soluzioni di alloggio elitarie ma è orientato al risparmio.

Al fine di delineare i profili di ciascun segmento, sono state predisposte delle tabelle a doppia entrata che mettessero in relazione le risposte degli appartenenti ai due segmenti con le domande del questionario. L'analisi 
sinergie

italian journal of management Vol. 35, N. 103, 2017

non ha evidenziato differenze nette per tutte le domande, ed in particolare la cultura e l'enogastronomia si confermano le principali motivazioni di viaggio in Sicilia, per gli appartenenti ad entrambi i cluster. I principali elementi di differenza tra i due segmenti riguardano l'età e l'organizzazione della vacanza. Con riferimento all'età, il segmento dei tradizionalisti è per il $69 \%$ costituito da soggetti con più di 44 anni, mentre il segmento dei moderni è per il 58\% composto da soggetti fino ai 44 anni. Questi dati socio-demografici sono coerenti con i comportamenti di viaggio dei due segmenti, infatti i tradizionalisti sono più propensi ad utilizzare strumenti di contatto tradizionali, come il telefono, e preferiscono affidarsi ad un tour operator per organizzare e prenotare la vacanza; i moderni, invece, mostrano una più spiccata preferenza per l'uso del web e privilegiano il "fai da te" per l'organizzazione del viaggio.La tabella seguente mostra gli ambiti comportamentali in cui sono state rilevate le principali differenze tra i cluster.

Tab. 6: Caratteristiche comportamentali dei cluster

\begin{tabular}{|l|l|l|}
\hline Comportamenti di viaggio & Tradizionalisti & Moderni \\
\hline Informazioni tramite web & $75,2 \%$ & $76 \%$ \\
\hline Informazioni tramite telefono o di persona & $40,9 \%$ & $35 \%$ \\
\hline Fai da te & $25,9 \%$ & $66 \%$ \\
\hline Tour operator & $68,8 \%$ & $26 \%$ \\
\hline Prenotazione tramite web & $20,5 \%$ & $61 \%$ \\
\hline Prenotazione tramite telefono o di persona & $60,2 \%$ & $29 \%$ \\
\hline Viaggia solo & $8,6 \%$ & $9 \%$ \\
\hline Viaggia in coppia & $61,2 \%$ & $63 \%$ \\
\hline Viaggia con la famiglia & $48,4 \%$ & $49 \%$ \\
\hline Viaggia con amici & $41,9 \%$ & $39 \%$ \\
\hline
\end{tabular}

Fonte: Ns. elaborazione

\section{Considerazioni conclusive, contributi e limiti della ricerca}

Il trend attuale della domanda turistica evidenzia una sempre più spiccata preferenza verso esperienze ad elevato coinvolgimento fisico ed emozionale che, proponendo attività soprattutto di evasione ed educazione, contribuiscano a rendere il viaggio memorabile. Pertanto, una maggiore attenzione verso l'implementazione di forme di turismo creativo (Richards, 2011), potrebbe generare opportunità competitive per la destinazione turistica. In tale direzione, è necessaria, da una parte, la conoscenza delle preferenze e delle aspettative dei turisti, dall'altra, la capacità degli operatori di progettare offerte specifiche e pacchetti che, valorizzando le risorse, le tradizioni e le tipicità del territorio, propongano una soddisfacente combinazione di servizi ed esperienze.

A tal proposito, l' indagine condotta ha consentito di analizzare le preferenze dei potenziali viaggiatori rispetto ad alcuni pacchetti turistici e alle diverse attività di tipo creativo proposte al loro interno. 
Il campione di riferimento ha individuato nella cultura e nell'enogastronomia i principali "motori" di un viaggio in Sicilia. Esso mostra di preferire i pacchetti che comprendono la più ampia varietà di esperienze (enogastronomiche, ludiche, sportive, culturali), e in particolare quelli con almeno un' esperienza di tipo creativo.

L'analisi di segmentazione ha evidenziato, inoltre, una differenza di preferenze legata all' età dei turisti. I meno giovani, cd. tradizionalisti, preferiscono rivolgersi ai tour operator e sono disposti a pagare di più per avere una vacanza che li coinvolga al massimo, senza avanzare pretese sulla categoria dell'alloggio. I più giovani, cd. moderni, invece, utilizzano soluzioni fai da te e cercano di risparmiare, pur preferendo alberghi di più elevata categoria.

Questa ricerca offre contributi di tipo sia teorico che pratico. In primo luogo essa arricchisce la letteratura sul turismo creativo, contribuendo a colmare il gap di conoscenza sulle preferenze e sui comportamenti d'acquisto rispetto alle diverse tipologie di esperienze creative. In secondo luogo, l'analisi empirica condotta ha consentito di ricavare l'utilità associata alle diverse componenti dei pacchetti proposti, di individuare il pacchetto turistico ideale e di segmentare i potenziali turisti rispetto ai comportamenti ed alle preferenze di tipo creativo.

Infine il lavoro, evidenziando le tipologie di esperienze preferite dai potenziali turisti con riferimento al territorio siciliano, offre indicazioni utili alle aziende operanti in ambito turistico su alcune scelte che potrebbero svilupparne la competitività: alle imprese turistiche che intendano arricchire e migliorare la propria offerta specifica, rendendola maggiormente attrattiva rispetto alle nuove esigenze manifestate dal mercato; ai tour operator, nella selezione delle esperienze per la composizione dei pacchetti creativi. In una prospettiva più ampia di destination management, la ricerca offre informazioni e stimoli ai policy maker affinché, nella formulazione di politiche di marketing territoriale, possano, da una parte, indirizzare lo sviluppo dell'offerta territoriale verso attività di tipo creativo, coinvolgendo in questa direzione le imprese e le organizzazioni attive sul territorio, dall'altra rivedere le scelte di posizionamento enfatizzando la componente creativa dell'offerta territoriale che rappresenta un fattore rilevante di attrattiva turistica.

Questa ricerca presenta tuttavia alcuni limiti, che suggeriscono lopportunità di ulteriori approfondimenti. Un primo limite è rappresentato dal fatto che l'indagine è stata condotta con riferimento ad una specifica area geografica di destinazione, quella siciliana. Un secondo limite risiede nel fatto che, al fine di non rendere troppo complessa l'analisi, si è scelto di articolare i differenti attributi che costituiscono i pacchetti turistici in soli tre livelli, supponendo, inoltre, un soggiorno non più lungo di quattro giorni.

Nuovi approfondimenti della ricerca potrebbero riguardare, da una parte, l'estensione dell'analisi ad altri contesti territoriali, al fine di comprendere quanto le preferenze espresse dai turisti siano influenzate dalle peculiarità dei territori, dall'altra, l'ampliamento dello spettro di attività creative inserite nei pacchetti sottoposti alla valutazione dei potenziali turisti.
Sonia Caterina Giaccone Marco Galvagno Elisa Di Mauro Competere attraverso il turismo creativo: unanalisi delle preferenze verso la Sicilia 


\section{sinergie}

Vol. 35, N. 103, 2017

\section{Bibliografia}

ALI F., RYU K., HUSSAIN K. (2016), "Influence of experiences on memories, satisfaction and behavioral intentions: A study of creative tourism", Journal of Travel and Tourism Marketing, vol. 33, n. 1, pp. 85-100.

BEERLI A., MARTIN J.D. (2004a), "Tourists' characteristics and the perceived image of tourist destinations: a quantitative analysis - a case study of Lanzarote, Spain", Tourism Management, vol. 25, n. 5, pp. 623-636.

BRUNETTI F. (1999), Il turismo sulla via della qualità, Cedam, Padova.

BUCHMANN A., MOORE K., FISHER D. (2010), "Experiencing film tourism: Authenticity \& fellowship", Annals of Tourism Research, vol. 37, n. 1, pp. 229-248.

CARBONE L.P., HAECKEL S.H. (1994), "Engineering customer experiences", Marketing Management, vol. 3, n. 3, pp. 8-19.

CHANG L.L., BACKMAN K.F., CHIH HUANG Y. (2014), "Creative tourism: a preliminary examination of creative tourists' motivation, experience, perceived value and revisit intention", International Journal of Culture, Tourism and Hospitality Research, vol. 8, n. 4, pp. 401-419.

COHEN E. (1993), “The heterogeneization of a tourist art”, Annals of Tourism Research, vol. 20, n. 1, pp. 138-163.

CREIGHTON M.R. (1995), "Japanese craft tourism: Liberating the crane wife", Annals of Tourism Research, vol. 22, n. 2, pp. 463-478.

CROCE E., PERRI G. (2008), Il turismo enogastronomico. Progettare, gestire, vivere l'integrazione tra cibo, viaggio, territorio, Franco Angeli, Milano.

DANIEL Y.P. (1996), "Tourism dance performances authenticity and creativity”, Annals of Tourism Research, vol. 23, n. 4, pp. 780-797.

FARIAS S.A, AGUIAR E.C., MELO F.V.S. (2014), "Store atmospherics and Experiential Marketing: a conceptual framework and research propositions for an extraordinary Customer experience", International Business Research, vol. 7, n. 2, pp. 87-99.

FERNANDES C. (2011), "Cultural planning and creative tourism in an emerging tourist destination", International Journal of Management Cases, vol. 13, n. 3, pp. 629-636.

FURLAN R., MARTONE D. (2011), La Conjoint analysis per la ricerca sociale e di marketing, Franco Angeli, Milano.

GARG R., RAHMAN Z., KUMAR I. (2010) "Evaluating a model for analysing methods used for measuring customer experience", Database Marketing and Customer Strategy Management, vol. 17, n. 2, pp. 78-90.

GENTILE C., SPILLER N., NOCI G. (2007), "How to sustain the Customer experience: an overview of experience components that co-create value with the customer", European Management Journal, vol. 25, n. 5, pp. 395410.

GIBSON C., CONELL J. (2005), Music and tourism: on the road again (vol. 19), Channel View Publications, Clevedon, UK.

GUPTA S., VAJIC M. (1999), "The contextual and dialectical nature of experiences" in Fitzsimmons J., Fitzsimmons M. (eds.), New service development, Thousand Oaks, , pp. 33-51, Sage, CA.

HUDSON S., RITCHIE J.B. (2006), "Film tourism and destination marketing: The case of Captain Corelli's Mandolin", Journal of Vacation Marketing, vol. 12, n. 3, pp. 256-268. 
HULL J.S., SASSENBERG U. (2012), "Creating new cultural visitor experiences on islands: Challenges and opportunities", Journal of Tourism Consumption and Practice, vol. 4, n. 2, pp. 91-110.

JONES D., SMITH K. (2005), "Middle earth meets New Zealand: Authenticity and location in the making of The Lord of the Rings", Journal of Management Studies, vol. 42, n. 5, pp. 923-945.

KIM J.H. (2010), "Determining the factors affecting the memorable nature of travel experiences", Journal of Travel and Tourism Marketing, vol. 27, n. 8, pp. 780-796.

KIM J.H. (2014), "The antecedents of memorable tourism experiences: The development of a scale to measure the destination attributes associated with memorable experiences", Tourism Management, n. 44, pp. 34-45.

KIM J.H., RITCHIE J.R., TUNG V.W.S. (2010), "The effect of memorable experience on behavioral intentions in tourism: A structural equation modeling approach", Tourism Analysis, vol. 15, n. 6, pp. 637-648.

LEWIS R.R., CHAMBERS R.E. (2000), Marketing leadership in hospitality, Foundations and Practices, $3^{\text {rd }}$ ed., Wiley, New York.

MAITAL S. (1999), "The Experience Economy: Work is theatre \& every business a stage”, Sloan Management Review, vol. 40, n. 4, pp. 93-95.

MORGAN M., WATSON P., HEMMINGTON N. (2008), "Drama in the dining rooms: Theatrical perspectives on the foodservice encounter", Journal of Foodservices, n. 19, pp. 111-118.

O'SULLIVAN E., SPANGLER K. (1998), Experience marketing: strategies for a new millennium, Venture Publishing, State College, PA.

OECD (2014), “Tourism and the Creative Economy”, OECD Studies on Tourism, OECD Publishing. http://dx.doi.org/10.1787/9789264207875-en

OH H., FIORE A.M., JEOUNG M. (2007), "Measuring experience economy concepts: tourism applications", Journal of Travel Research, vol. 46, n. 2, pp. 119-132.

PEARCE D.G., BUTLER R.W. (1993), Tourism research: Critiques and challenges, Routledge, London.

PENCARELLI T., FORLANI F. (2011), "Il marketing dei distretti turistici-sistemi vitali nell'economia delle esperienze", Sinergie, n. 58, pp. 232-277.

PINE B.J., GILMORE J.H. (1998), "Welcome to the experience economy", Harvard Business Review, vol. 76, n. 4, pp. 97-105.

PINE B.J., GILMORE J.H. (2000), Leconomia delle esperienze, Etas, Milano.

PRAHALAD C.K., RAMASWAMY V. (2000), "Co-opting customer competence", Harvard Business Review, vol. 78, n. 1, pp. 79-90.

PREBENSEN N.K., FOSS L. (2011), "Coping and co-creating in tourist experiences”, International Journal of Tourism Research, vol. 13, n. 1, pp. 54-67.

RAYMOND C. (2007), "Creative tourism New Zealand: The practical challenges of developing creative tourism", in Richards G. and Wilson J. (Eds.), Tourism, Creativity and Development, Routledge, London, pp. 145-157.

RICHARDS G. (2000), “Tourism and the World of Culture and Heritage”, Tourism Recreation Research, vol. 25, n. 1, pp. 1-31.

RICHARDS G. (2011), "Creativity and Tourism- The State of art", Annals of Tourism Research, vol. 38, n. 4, pp. 1225-1253.

RICHARDS G., RAYMOND C. (2000), “Creative Tourism”, ATLAS News, n. 23, pp. 16-20.
Sonia Caterina Giaccone

Marco Galvagno

Elisa Di Mauro

Competere attraverso

il turismo creativo: unanalisi

delle preferenze verso

la Sicilia 
RICHARDS G., WILSON J. (2006), “Developing creativity in tourist experiences: a solution to the serial reproduction of culture?", Tourism Management, vol. 27, n. 6, pp. 1209-1223.

SWAIN M.B. (1993), "Women producers of ethnic arts", Annals of Tourism Research, vol. 20, n. 1, pp. 32-51.

TAN S.K., KUNG S.F., LUH D.B. (2013), "A model of 'creative experience'in creative tourism", Annals of Tourism Research, n. 41, pp. 153-174.

TAN S.K., LUH D.B. KUNG S.F. (2014), “A taxonomy of creative tourists in creative tourism”, Tourism Management, vol. 42, pp. 248-259.

TRIPBAROMETER (2014), "Psicologia del viaggiatore - Rendiconto globale", IPSOS.

TUNG V.W.S., RITCHIE J.R.B. (2011), "Exploring the essence of memorable tourism experiences", Annals of Tourism Research, vol. 38, n. 4, pp. 13671386.

VERHOEF C., LEMON K.N., PARASURAMAN A., ROGGEVEEN A., TSIROS M., SCHLESINGER L.A. (2009), "Customer experience Creation: Determinants, Dynamics and Management Strategies", Journal of Retailing, vol. 85, n. 1, pp. 31-41.

WALLS A.R., OKUMUS F., WANG Y.R., KWUN D.J.W. (2011), “An epistemological view of consumer experiences", International Journal of Hospitality Management, vol. 30, n. 1, pp. 10-21.

\section{Academic or professional position and contacts}

Sonia Caterina Giaccone

Researcher of Management

University of Catania - Italy

e-mail:s.giaccone@unict.it

\section{Marco Galvagno}

Associate Professor of Management

University of Catania - Italy

e-mail: mgalvagno@unict.it

\section{Elisa Di Mauro}

M.Sc. in Management

University of Catania - Italy

e-mail: elydimauro@virgilio.it 